\section{A TRUE ALL-IN-ONE SYSTEM}

Bien-Air has developed probably the most efficient dental micromotor on the market with the Optima MX2 INT.

Thanks to Smart Logic technology, the Optima MX2 INT control unit offers ultimate regulation of the power required, linearly and without vibration, ensuring you are perfectly in control of the speed, torque and reversal at all times. With Easy-Nav philosophy the Optima MX2 INT is incredibly intuitive and adapts to most dental chairs. The control unit offers ten pre-programmed modes for your main restorative work and ten sequences for your endodontic work, all at the press of a button.

This Swiss-made control unit has been developed with your endodontic work in mind. You can perform your root treatments with complete peace of mind as the Optima MX2 INT has been designed to reduce the risk of NiTi instrument breakage to an absolute minimum with the precise auto reverse function.

A true all-in-one system, the Optima MX2 INT makes your life easier. In most cases two contra-angle handpieces are sufficient to carry out the vast majority of dentistry.

For further information contact Bien-Air
OPTIMA MX2 Endo file ${ }^{*}$ Ratio $1: 1$ 250 rem Torque 1.01nca $\Theta \Theta \Theta \Theta$

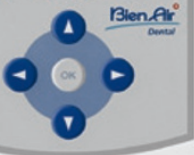
on 01293 550200 or visit wWw. bienair.com.

\section{DUST AND MIST-FREE LIGHT} sensor to turn the light on/off as well as allowing the user to switch to a composite cure mode setting so that you can work uninterrupted with compositecure materials. As the ten LED lights are encased in a one piece cover there's no risk of dust or mist build-up and the unit is easily cleaned.
Takara Belmont's LED light unit has a touchless

\section{DENTISTS WANTED IN THE ARMY NOW}

The British Army is looking to hire dentists as part of the latest recruitment effort to bring 10,000 new soldiers and officers into the Army over the coming year.

The 'Step Up' campaign aims to dispel the misconception that the Army is not hiring as new statistics reveal that only half of all people surveyed realised that the Army is looking for new recruits.

There are over 200 different specialist roles available with the Army including careers for specialists such as dentists.

Life with the Royal Army Dental Corps (RADC) offers a great deal more than team work and achieving high professional standards. There are also plenty of opportunities for sports, adventure training, social clubs and associations with all other Corps and Regiments within the Army.

Whichever RADC trade a soldier adopts, it provides a full and rewarding career with excellent training, standing him or her in good stead when the time comes to return to civilian life.

For more information visit www.army.mod.uk/Join/ StepUp/boots.aspx or call 08456008080.

\section{CASTING ALLOYS INTELLIGENTLY}

BEGO has revised its Nautilus machines for casting dental alloys intelligently. Nautilus CC plus and Nautilus T now have integrated, water-independent power cooling which makes it possible to perform 50 casting procedures in sequence without overheating - even in high ambient temperatures. The machines are operated and controlled using an intuitive touch screen, accelerating the access times. The new eco-mode is particularly economical. In addition, the floor space required with

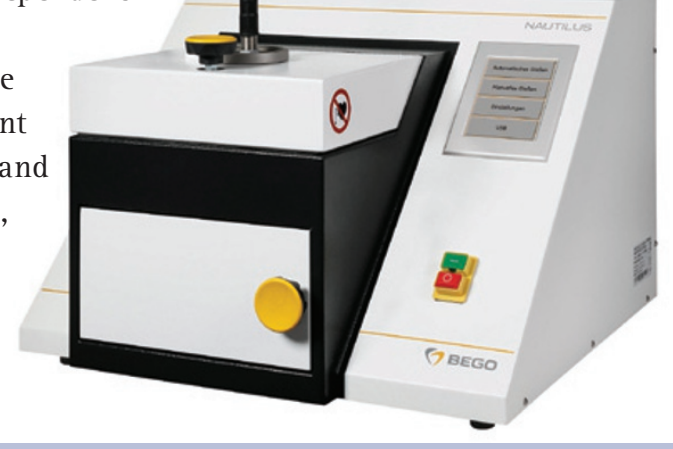

\section{INDUSTRY-LEADING IMAGE RESOLUTION}

Schick 33 from Clark Dental has an industry-leading image resolution of 33 line pairs per millimetre.

Thanks to the modular nature of the Schick Digital Imaging System, if you already own Schick products, upgrading is simple, and when further updates become available, you can upgrade further.
With the incredible power of Schick 33 you really can see things your way. Schick 33 allows you to change your settings according to your specific diagnostic needs. For instance, use Schick 33's clinical-specific mapping feature to automatically default to presets for general dentistry, endodontics, periodontics or restorative dentistry. Or, create even more personal 'me-sets' for you and your colleagues that can be easily saved and shared across your practice.

Call 01268733 146. www.clarkdental.co.uk over a conventional bulb. With a projected lifetime of a staggering 40,000 hours or around 25 years, dental professionals will know that this equipment will carry them through the majority of their career.

For further information call 02075150333.
The LED 900 light offers many dvantages over a traditional halmaking it ideal for colour matching and reducing eye fatigue. They also give out less heat and 